\title{
Different Futures of Adaptive Collaborative Learning Support
}

\author{
Nikol Rummel $^{1,2} \cdot$ Erin Walker $^{3}$ - Vincent Aleven ${ }^{2}$ \\ Published online: 22 February 2016 \\ (C) International Artificial Intelligence in Education Society 2016
}

\begin{abstract}
In this position paper we contrast a Dystopian view of the future of adaptive collaborative learning support (ACLS) with a Utopian scenario that - due to betterdesigned technology, grounded in research - avoids the pitfalls of the Dystopian version and paints a positive picture of the practice of computer-supported collaborative learning 25 years from now. We discuss research that we see as important in working towards a Utopian future in the next 25 years. In particular, we see a need to work towards a comprehensive instructional framework building on educational theory. This framework will allow us to provide nuanced and flexible (i.e. intelligent) ACLS to collaborative learners - the type of support we sketch in our Utopian scenario.
\end{abstract}

Keywords Computer-supported collaborative learning - Adaptive collaborative learning support · Theory of collaborative learning · Instructional theory

\section{Introduction}

The present paper focuses on an area where computer-supported collaborative learning (CSCL) and AIED research intersect: adaptive collaborative learning support (ACLS, see Walker et al. 2009b). This area has recently received increasing attention, as evidenced, for example, by the 2014 IJAIED special issue entitled "Intelligent Support for Learning in Groups" (ISLG), as well as by the conference workshop series of the

Nikol Rummel

nikol.rummel@rub.de

Erin Walker

erin.a.walker@asu.edu

1 Institute of Educational Research, Ruhr-Universität Bochum, Bochum, Germany

2 Human-Computer Interaction Institute, Carnegie Mellon University, Pittsburgh, PA, USA

3 School of Computing, Informatics, and Decision Systems Engineering, Arizona State University, Tempe, AZ, USA 
same name at recent AIED and ITS conferences. ACLS involves the use of intelligent technologies to improve student collaboration and learning by assessing the current state of the interaction and providing a tailored pedagogical intervention (Soller et al. 2005). ACLS differs from typical intelligent tutoring in that its goals are to improve the collaboration between two or more students, rather than to support the learning of an individual student. In recent years, it has proven to be a promising method of supporting CSCL in a way that caters to the needs of particular groups of students, with benefits compared to individual work, collaboration with no support, and collaboration with non-adaptive support (Baghaei et al. 2007; Kumar et al. 2007; Walker et al. 2014). ACLS support can focus on group formation, on supporting domain knowledge, or on improving peer interaction (Magnisalis et al. 2011). To produce this support, a range of natural language processing, machine learning, and user modelling techniques are used. For example, the APTA system (Walker et al. 2014) provides peer tutors with reflective prompts to improve both the quality and content of their actions. It uses an integrated cognitive model and machine classifier to diagnose both domain errors made by a peer tutor and ineffective tutoring approaches (Walker et al. 2014).

In this paper, we consider two possible futures for the area of adaptive support for collaborative learning. First, we sketch a scenario that illustrates where ACLS might be 25 years down the road if technological solutions are not informed by educational theory and research. It is a scenario that we view as Dystopian, because the full potential that we see for adaptive and adaptable technologies has not been fully realized. It is not a desirable scenario, but in our opinion a possible one. As a contrast we present a more optimistic, Utopian, scenario, one in which learners are empowered for collaborative learning by flexible, adaptive support that avoids the pitfalls of the Dystopian scenario. The Utopian scenario requires a challenging research agenda for the next 25 years. We pose that working towards a comprehensive instructional framework for ACLS can help to prevent the Dystopian and pave the way for a Utopian future of ACLS.

In this paper we focus on collaboration in small groups of learners working together side bv side (i.e. face to face). The envisioned instructional framework also speaks to collaborative learning in computer-mediated settings as well as to learning in large groups, although there the picture becomes even more complex due to the number of actors involved.

\section{Fast-Forward to the Year 2040: a Dystopian Scenario}

Janet got to school, and ran to her first period class. Chemistry. 5 min to spare. Perfect. She looked down at her digital organizer to see who she was assigned to today. Roxanne. Crap. She'd had Roxanne three times in the past two weeks, and each time, they ended up arguing heatedly about one thing or another - is it a molecule or an atom? Should they titrate or just leave it? Janet knew that last time Roxanne had asked their teacher, Mr. Roebeck, to be assigned a different partner. Roxanne reported back that Mr. Roebeck had said that the system was pairing the two of them because they "experienced constructive conflict." Janet thought that personally, she could do with a little less conflict and could not quite see how the frustrating experience with Roxanne could be constructive. She always had fun working with Carly, they seemed to understand each others' explanations and could easily agree on a way forward! But, as the technology director of the school always said, "RUWAAL knows best." 
RUWAAL was the ACLS system implemented by the school, and there was no way to argue against the choices that it made.

"Ok," Mr. Roebeck said, "get in your pairs and check your prescribed activity." Janet reluctantly walked over to Roxanne and sat in the chair beside her. They looked down at their digital desk to see which lab activity was prescribed. They were supposed to figure out which functional groups were present in an organic molecule presented to them in $3 \mathrm{D}$ on their screen. As they worked, the system continuously prompted, "Janet, can you explain your reasoning?" "Janet, what do you think about what Roxanne just said?" RUWAAL understood all their speech and actions, and its collaborative model could respond instantaneously to guide them in the right direction. Janet found her mind wandering, though. She knew that the other students did not get nearly so many prompts. A while back, RUWAAL had diagnosed her as a "poor collaborator." Mr. Roebeck had told her parents that she would be getting remediation, but RUWAAL did not think that she would ever be able to work well with others. Her parents had an appeal out - they were outraged, but Janet was more confused by the situation than anything else. She enjoyed working with others - well, almost everyone except for Roxanne. The RUWAAL system was so sophisticated it could predict with $99 \%$ accuracy who should work with whom, what activities they should receive, what prompts they should get, to maximize learning. But the technology could not explain its decisions or provide useful advice! At least, Janet did not understand what the heck she was supposed to do to improve the way she worked in groups!

Janet's train of thought was interrupted by Roxanne's teasing voice. "Pay attention dummy! Look at what I just did!" The solution was now brightly colored. Janet stared at it, briefly fascinated. "I wonder why it did that?!" Roxanne said, "I do not know, that's pretty cool, right? "I don't know, Roxanne. I don't think that's part of the assignment." Janet thought about it. Roxanne was always goofing around, and sometimes it got them into trouble. RUWAAL agreed, by digitally resetting the solution to its original color. "Incorrect action. Try again. Follow the steps of the prescribed activity." "See, I told you!" said Janet. The system added, "Remember, it's important to be polite and kind to each other." It had picked up on Janet's mocking tone of voice, and was trying to calm her and Roxanne down. As usual.

Janet made an attempt to get the two of them back on track. She suggested different options for how they could move ahead to Roxanne and elaborated a bit on each of them to make sure that Roxanne would understand the ideas she put forward. She was just about to ask Roxanne to tell her what she thought of her ideas when RUWAAL interrupted Janet saying, "Remember that collaboration is about solving the problem together, not one telling the other what to do." Roxanne sneered. "Wanna hear my opinion?", she said in a taunting voice. "Well done, speak up and contribute to the collaboration", RUWAAL encouraged her.

Janet felt distracted, annoyed. Sometimes she thought things must have been better in the old days that her mother was always talking about, before RUWAAL, when students could just do what they wanted without the technology interfering and following every step they did. At that very moment, the system popped up an alert saying, "Janet, time to enter your next diary entry. Today, please reflect on how you are progressing as a collaborative learner." 


\section{Unpacking the Dystopian Scenario}

In our Dystopian vision 25 years into the future of AIED research, collaborative learning technologies are using the full capabilities that modern computing technology has to offer, such as near-perfect natural language understanding and recognition of emotion and motivation based on a host of variables, such as problem-solving actions and the content of student verbal utterances, but also prosodic cues, gaze patterns, physiological measures, gesture, and posture. If this Dystopian scenario seems unlikely, we view it as one possible straight-line extension of what is currently occurring in CSCL and educational technology research, with advances in text mining, speech processing, and gesture recognition, and analyses of large data sets to find links between collaborative process and outcomes. With increasing access to various data from collaborative learning processes and outcomes, it will likely be possible, in 25 years hence, to predict with great accuracy who will be successful in what collaborative learning setting and given what collaborative activity. The range of pedagogical decisions that is supported by or taken over by technology will likely expand compared to what is available today: automatic group formation, selection of activities for each group, and adaptive prompting and scripting to ensure learners are collaborating and learning effectively.

Nevertheless, there is much that is unsatisfying about this scenario. The system makes decisions about what will lead Janet to learn most effectively based on data about her, and other students', current and past activities, but these decisions lack nuance and flexibility, leading to frustration and ultimately to a lack of trust in the system. For instance, the system is unable to distinguish between constructive conflict and conflict that diminishes student motivation and prevents them from learning. Thus the system continues to pair Janet with Roxanne based on the characteristics of previous pairs that have been successful in its database: In previous pairings, those characteristics were a predictor of a certain level of conflict, and this conflict was a predictor of learning success in the end. While Janet and Roxanne do experience conflict, the conflict decreases Janet's motivation. Janet and Roxanne continue to clash, but fail to move ahead in solving the problem ahead and learning from their joint work.

A key shortcoming of RUWAAL is that the different dimensions of support in its ACLS are not well coordinated and thus are not working together in a coherent, holistic way: support is always given immediately, directed at the person whose action triggered the system reaction, focused on the psychological realm where a need for support was diagnosed (i.e. cognitive, social, motivational) but with a strong emphasis on cognitive support, and presented in a guiding fashion. A more Utopian system would be able to orchestrate the different dimensions of support (i.e., timing of support, psychological realm, mode and locus of support, and support type) in nuanced ways. For instance, based on a careful analysis of the current situation and taking into account similar conflict in the pairs' past, RUWAAL might have decided to have the two girls engage in some other collaborative activity first, to support team building, before putting them in front of their next collaborative Chemistry task.

A related shortcoming evident from the Dystopian scenario is that the system's pedagogy is very limited. For example, the system does almost nothing to help students work through disagreement productively. It accurately "sensed" Janet's frustration, but its response (urging politeness) was a "local patch" rather than a nuanced strategy that 
viewed the causes of her frustration in light of the goals of the assignment and Janet's and her partner's active learning goals.

A final problem is that RUWAAL's decisions are inscrutable to learners and teachers. Janet has trouble understanding the system's pairing decisions and is increasingly reacting negatively towards them. However, instead of offering her help in understanding its decisions or giving her choices, the system tries to keep Janet "on track". Similarly, the teacher is relegated to trying to justify the system's decisions, rather than being empowered to make his own choices with the system's active assistance. To summarize, we view the above scenario as Dystopian because the futuristic ACLS system is theoretically and pedagogically limited, does not coordinate across different dimensions of support, and does not make decisions that are transparent to and adaptable by students and teachers.

\section{A Contrasting View: a Utopian Scenario for 2040}

Janet woke up looking forward to what the school day would bring: collaborative inquiry in chemistry lab, with guidance from RUWAAL! This year was the first year that many learning activities in her grade level were done collaboratively. After a bumpy start, she had really gotten the hang of it. She enjoyed the lively back-andforth with a collaborative partner, as she had told RUWAAL during one of her selfratings of the collaborative sessions. She expected that today's activities would be challenging, but she liked to be challenged and she felt that RUWAAL had helped her grow, both in learning chemistry and in learning to be a good collaborator.

In the early weeks of the semester, RUWAAL had pushed Janet to speak more during her collaborative activities, which, although uncomfortable at first (RUWAAL seemed to understand, though) had really helped her. A highlight for Janet was a review session with RUWAAL a few weeks ago, in which it presented stats showing that as she had started to talk more, her partners did the same, often resulting in good discussions about challenging chemistry concepts. RUWAAL also replayed two brief clips from Janet's activities: The first, from early in the semester, showed her attentively listening to Roxanne's explanation of the concept of chemical equilibrium. The second, from two months later, showed her initiating an exchange with Roxanne by saying: "Hmmm... dynamic equilibrium is when things change but there is no net change? Not sure I get that. Let me try to talk this through, and perhaps you can help me fill in the gaps ..." From RUWAAL's point of view, Janet's increased comfort level with formulating even half-baked thoughts as a way of learning was evident in a number of ways: her increased contributions in the dialogue, the fact that she stepped into the fray earlier and more often, and the more searching tone of her voice followed by increments in understanding, based on RUWAAL's content analysis of the speech signal. These inferences caused RUWAAL to increase its estimates of the trust level between the students. RUWAAL passed this information on to Janet's teacher, Ms. Holzenbein, who made sure to encourage Janet to continue to speak up when uncertain.

Janet read her assignment for the day: explore the Law of Mass Action with Isabelle ... wow, challenging! New material, a new simulator, and a partner with whom she had not worked before. Although Janet did not know this, RUWAAL had selected 
this assignment in consultation with Ms. Holzenbein. Specifically, RUWAAL had offered Ms. Holzenbein a choice of two alternative assignments for Janet to work on next: the one with Isabelle, and one together with Roxanne, Janet's frequent partner, a review lesson that would be the typical next step for them. RUWAAL explained to Ms. Holzenbein that the first of these assignments likely would be more challenging for Janet. It suggested that Ms. Holzenbein consider whether Janet was ready for a tough challenge right away or whether Janet might be better off if that challenge came after the weekend. RUWAAL also explained that if Janet worked with a new partner, she could take the next step in becoming a good collaborative learner. RUWAAL knew that, at that point in time, Janet was a better collaborative learner than Isabelle. It knew also that she had stronger chemistry knowledge, so it was likely that some frustration might occur. Ms. Holzenbein however had noticed Janet's eagerness to be challenged and her increased comfort with collaborative learning, so selected the first option for Janet's assignment. Being consulted on this kind of choice was according to Ms. Holzenbein's preferences, told to RUWAAL at the beginning of the semester.

Janet and Isabelle started off well enough, but then hit an impasse, which, in spite of much discussion and many solution attempts, they were not able to get past. RUWAAL noticed the stagnation and frustration, and considered how to step in. Should it try to get the students past the impasse by promoting domain-level success, for example, by providing a domain-level hint or even by giving the next step? Or should it try to help the students collaboratively work through the impasse, which might help them become better collaborative learners and enable them to deal with similar situations independently in the future? In the given situation, RUWAAL judged the frustration level to be relatively high. Although that might have been reason to provide domain-relevant advice, so as to resolve the impasse quickly, it decided to focus instead on its goal to help Janet take the next step in becoming a good collaborative learner. It led off with a decompress strategy. "Hey guys, I know you are into this a lot, you might not be far away from the solution, but why don't you take a brief break? The cafeteria has new coconut macaroons!" Janet and Isabelle had a good time in the cafeteria. When they came back, RUWAAL, noticing their improved mood, said, "Did you know macaroons are chemistry, too? Now let us get back to work." After some lively discussion, soon Janet and Isabelle were moving forward again. Isabelle said to Janet: "Hey, good session! I like working with you." That evening, Janet figured that she had learned a good way of managing frustration in a collaborative partnership. RUWAAL bookmarked the episode for later use in Janet's and Isabelle's periodic reviews, and set an instructional goal to have Janet and Isabelle learn other strategies for dealing with frustration.

\section{Unpacking the Utopian Scenario}

The Utopian scenario highlights positive outcomes that ACLS will have in 2040 if the research outlined below is successful. The ACLS system of the Utopian scenario is different in many ways from that of the Dystopian scenario presented earlier. Its decision making is driven by theory, takes into account a variety of pedagogical goals (including learning at the domain level and learning of collaboration skills), and draws on a wide arsenal of pedagogical strategies. It coordinates support across multiple 
dimensions (e.g., timing of support, psychological realm of support, mode of support, and support type), enabling it to flexibly adapt to students' needs. Finally, the system is adaptable: It is transparent, works synergistically with students and teachers, and shares control with them.

The ACLS system's balanced theory-driven integration of multiple dimensions of support for collaborative learning is on display when our student (Janet) spontaneously starts to self-explain a difficult chemistry concept. Anticipating she has some missing knowledge, she right away invites feedback from her partner. This is a remarkably sophisticated collaborative learning behaviour for a 15-year old. RUWAAL recognized it as such, helped by what it knows about the two students individually as well as their past collaborations, and other comparable students' collaborations on similar tasks. It noticed that Janet had a learning goal of better understanding the given chemistry concept, was able to self-assess her knowledge, and choose an appropriate learning strategy. In terms of collaboration, it recognized her statement to her partner as a form of proactive help seeking, even before the help was actually needed, as was appropriate in light of the chosen strategy. It also interpreted this move as indicating a high level of trust in her partner, consistent with their past interactions. By integrating these different perspectives, the system was able to highlight Janet's move in her later review as indicative of a positive trend in how Janet approached collaborative learning tasks. The integration helped avoid the disjointedness of support that was a key problem in the Dystopian scenario, resulting in a much more purposeful intervention.

This integration carries through to the instructional techniques the ACLS system uses to improve Janet's collaborative experience. It provides students with adaptive prompts and supports group formation, but unlike the Dystopian system, it balances that with providing reflective guidance to students and teachers. In choosing possible next tasks for students, the Utopian system takes into account goals for domain-level learning as well as goals for the learning of collaborative skills, and selects a partner who may help in both regards. It considers some of the same factors in other pedagogical decisions, such as how to recover from a frustrating impasse. In making this decision, the system considers different pedagogical moves, such as whether the impasse could be addressed at the domain level or at the collaboration level, and how the impasse interacts with social factors. Based on these factors, RUWAAL decides it will suggest that the students take a brief break, which turns out to be a successful strategy.

The scenario also illustrates the importance of creating technology that is adaptable. The system defers to the teacher regarding such intangible decisions as whether the time is right for upping the challenge level for a given team of students. The scenario illustrates how CSCL systems can leverage transparency regarding their assessment of learners and their decision making, leading to trust rather than alienation, of which there was far too much in the Dystopian scenario. Although this is not apparent at the surface, a fundamental reason the system is able to do so is because its underlying models are explainable models. These models are made possible, in part, by grounding the system in a comprehensive instructional framework, discussed below.

All this adds up to a more positive experience with better outcomes. While we have labelled the scenario as Utopian, we think of it as an optimistic but possible future for 2040, not Utopian in the sense of representing an unrealistic future we can only dream of. 


\section{A Research Agenda for 2016-2040}

The differences between our two scenarios exemplify the dilemmas that designers of ACLS systems face. The kind of coordinated pedagogical decision making illustrated in the Utopian scenario is a tough balancing act, even for humans, but one that the area of ACLS will need to tackle. A key shortcoming of the ACLS system sketched in the Dystopian scenario is that it does not have an elaborate theoretical foundation for its decisions. Taking into account the issues identified in unpacking the Dystopian scenario, we see a need to work towards a comprehensive instructional framework firmly rooted in educational theory that can guide the development of nuanced and flexible ACLS systems. These systems take into account multiple dimensions of support and balance system adaptivity with user freedom and shared user/system control.

The proposed research can build on prior work in ACLS, which has produced several taxonomies of support for collaborating students. These taxonomies (e.g. Diziol and Rummel 2010; Walker et al. 2009a) identify dimensions such as the timing of support (whether it is provided immediately or with some delay during the collaboration, or before or after the collaboration), the psychological realm of support (cognitive, social, metacognitive, motivational, affective), the mode of support (explicit or implicit), the locus of support (direct or indirect), the target of support (group formation, domain knowledge, peer interaction, social skill; Magnisalis et al. 2011), and the type of support (guiding, challenging reflection, mirroring; Soller et al. 2005). While these taxonomies describe different properties of support, they fall short of providing an integrated instructional framework that builds on learning theory and instructional design principles. Such a framework is needed to orchestrate support across the multiple dimensions, thus arriving at nuanced ACLS. The main work to be done by 2040 is to create this kind of framework for ACLS.

To move towards the aspired instructional framework, we must carry out rigorous empirical research and engage in related theory-building efforts. First, we need research within each individual support dimension. For instance, the timing of support dimension taps into the so-called assistance dilemma (Koedinger and Aleven 2007), which poses the fundamental question of how to balance the giving and withholding of assistance to achieve optimal student learning. Whereas in some cases providing immediate assistance may serve as a scaffold, in other cases it may be a crutch that prevents students from engaging in sense-making activities on their own and acquiring deep knowledge. Whereas withholding assistance might in some cases lead students to struggle and experience extraneous cognitive load, in other cases it may create desirable difficulties that enables students to learn by overcoming challenges (Koedinger et al. 2008). Similar constraints apply to CSCL environments, where there is evidence that delaying support may lead to productive learning conditions (e.g. Kapur 2008; Kapur and Kinzer 2009). Similarly, fading support over time (Fischer et al. 2013; Wecker and Fischer 2007), and adapting support based on student collaborative skill (Walker et al. 2014) have been shown to be effective. However, we do not yet have empirically - validated instructional principles that help decide when and how much support to give to collaborating students in any given context.

Secondly, let us consider the dimension of mode of support. Even if the support is given at opportune times, there is still no guarantee that students will pay attention to it. 
For example, Kumar et al. (2007) found that students tended to ignore adaptive prompts while collaborating. It might be that students ignore explicit adaptive feedback when it appears irrelevant to their task or violates other Gricean maxims (Bernsen et al. 1997). If the feedback is perceived as intrusive and critical, it might also threaten their sense of control (Nicol and Macfarlane-Dick 2006), or disrupt their belief that interpersonal risk taking is safe in a collaborative context, an important contributor to effective team learning behaviors (Van den Bossche et al. 2006). It is necessary that any support given is either sufficiently implicit as to not interrupt student activity (e.g., adaptive task selection) or highly socially sensitive to the collaborators' conversations. Further research on this dimension is needed to develop an understanding of what makes learners accepting of ACLS so as to be able to design support that learners will want to use.

A third substantial challenge is to decide on the psychological realm of support to be targeted in given circumstances. Several realms can be targeted, beyond domain-level support: for instance, metacognitive support, motivational support, and support at the social level (e.g., Muldner et al. 2010; Ogan et al. 2010; Roll et al. 2011). It is difficult to know which psychological realm to address, or how to combine support targeted at different realms. When deciding how to provide support in CSCL environments, it is fruitful to consider these multiple realms simultaneously. For example, when deciding (on the fly, based on the dynamics of the given collaborative learning situation) how much support to give regarding domain-level concepts or skills (i.e. cognitive support), it is helpful to take into account needs for motivational and social support. For example, in the Utopian scenario the system decides to provide motivational and social support, because this combination may further the goal of helping students progress on the domain level.

In addition to investigating how best to design and deliver support for each individual dimension of ACLS (timing, realm, mode, locus, presentation target, and type of support), we need to work towards coordinated decision-making across the dimensions. The large array of possibilities that arises from combining the various dimensions poses a significant challenge for research in the field of ACLS. This challenge will likely keep the field busy for the next 25 years and beyond. For instance, taking into account just the two dimensions mode of support (i.e. whether the action that students should take is explicitly described in the feedback or arises implicitly as a result of the support) and locus of support (i.e., whether the support is presented as directly addressing the person it targets or presented indirectly to another party or through a change in the learning environment) opens up a number of different ways ACLS can be delivered (Fig. 1; see Walker et al. 2009a): In many existing ACLS approaches, modelled after individual intelligent tutoring systems, support is explicit and direct (see lower right quadrant of Fig. 1). However, as illustrated in the Figure, several other possibilities for how to deliver ACLS arise when exploring the quadrants of the combination space. Peer-Mediated Feedback (the bottom-left quadrant) encourages collaborating partners to co-regulate their learning. For example, if one student is not explaining a step clearly, we can prompt their partner to ask, "What do you mean by that?" rather than asking the first student to expand their explanation. This approach is indirect, as the feedback does not directly address the relevant student, and it is explicit because the next course of action is clear. Adaptive Resources (the top-right quadrant) are resources provided to students at moments when 
they need them. For example, a video related to a given concept could be presented when a student may be thinking of applying the concept. The resource is directed to the relevant student, but the course of action suggested is implicit. Adaptive Opportunities (top-left quadrant) modify the learning environment in order to create learning opportunities for students, for example, by assigning problems adaptively to students based on their previous interactions. Here, the change to the learning path is implicit, and feedback is presented indirectly.

This example illustrates possibilities for combining of two dimensions of support. At this point in time, we do not know a principled way of choosing from among these possibilities, however, in any given learning context. Further, in order to arrive at the nuanced support illustrated in the Utopian scenario, decisions about support ought to be coordinated across all possible dimensions. This will only be possible if we work towards a comprehensive instructional framework of ACLS in the next years.

A further desirable aspect of the aspired instructional framework is to allow us to better balance system adaptivity and feedback with user choice and freedom. A focus on optimizing adaptivity of ACLS runs the risk of overemphasizing the role of the system in guiding the students, rather than empowering students and teachers to make good pedagogical choices with the help of the system. More research effort should be dedicated to the adaptability of ACLS systems (i.e. the possibility for users to adjust the collaborative learning situation to their current needs or goals by making active choices). There is some research on how to design CSCL systems that enable users to adapt them to their needs. For instance, mirroring systems that present to students visualizations of their collaborative activities enable students to use the visualizations to make their own adjustments to their collaborative activities (Soller et al. 2005). In another example, it has been investigated how collaboration scripts may be used to shift from system-regulation to self-regulation of a group (i.e. co-regulation of the learners) by fading out the script and transferring control to the learners within a

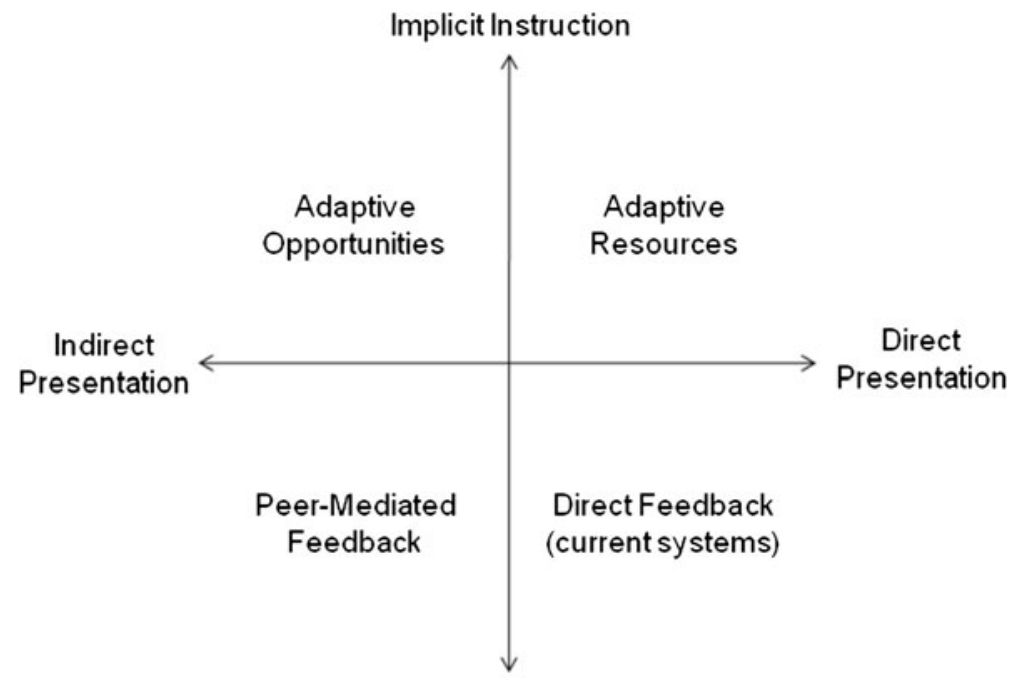

\section{Explicit Instruction}

Fig. 1 Design space for adaptive collaborative learning support (taken from Walker et al. 2009a). 
group who continue monitoring each other's activities (Wecker and Fischer 2011). More recently, first attempts have been made to make collaboration scripts adaptable, for example, to enable teachers and learners build their own scripts with the help of interoperable script components, (e.g. Prieto et al. 2012)., Adaptability by users is an area where further research is needed in the future.

\section{Conclusion}

Our vision for creating empowering ACLS in 2040 centers on the development of a comprehensive instructional framework that integrates theory of how people learn by collaborating, instructional theory of how to support collaboration, and instructional design principles based on rigorous empirical research. The instructional framework we envision will be detailed and precise enough to facilitate the implementation of nuanced, highly adaptive support in educational software. Development of this framework encompasses three components:

1. The integration of theories of collaborative learning to create a comprehensive framework that, alongside data-driven models, can inform support;

2. The derivation of principles of when, how, and what support to provide, which integrate the dimensions of support, so that decisions can be made in a coordinated and transparent manner;

3. The derivation of methods for balancing adaptivity and adaptability within support.

The research agenda we are advocating is a challenging one, because of the multidimensional nature of the support space mapped out above. It seems likely that in 2040, we will not be all the way to having a comprehensive and actionable instructional framework, but substantial progress is possible and necessary to avoid the problems introduced in the Dystopian scenario. If we are successful, the future will be like our Utopian scenario, where ACLS empowers students and teachers to make choices in learning contexts that foster promotive collaborative interactions and enhance the outcomes of collaborative learning activities.

\section{References}

Baghaei, N., Mitrovic, A., \& Irwin, W. (2007). Supporting collaborative learning and problem solving in a constraint-based CSCL environment for UML class diagrams. International Journal of ComputerSupported Collaborative Learning, 2(2-3), 159-190.

Bernsen, N. O., Dybkjær, H., \& Dybkjær, L. (1997). What should your speech system say? Computer, 30(12), 25-31. doi:10.1109/2.642788.

Diziol, D., \& Rummel, N. (2010). How to design support for collaborative e-learning: a framework of relevant dimensions. In B. Ertl (Ed.), E-collaborative knowledge construction: learning from computer-supported and virtual environments (pp. 162-179). Hershey, PA: IGI Global.

Fischer, F., Kollar, I., Stegmann, K., \& Wecker, C. (2013). Toward a script theory of guidance in computer-supported collaborative learning. Educational Psychologist, 48(1), 56-66. doi:10.1080/ 00461520.2012 .748005 .

Kapur, M. (2008). Productive failure. Cognition and Instruction, 26(3), 379-424. 
Kapur, M., \& Kinzer, C. (2009). Productive failure in CSCL groups. International Journal of ComputerSupported Collaborative Learning, 4(1), 21-46.

Koedinger, K. R., \& Aleven, V. (2007). Exploring the assistance dilemma in experiments with cognitive tutors. Educational Psychology Review, 19(3), 239-264.

Koedinger, K. R., Pavlik Jr. P. I., McLaren, B. M., \& Aleven, V. (2008). Is it better to give than to receive? The assistance dilemma as a fundamental unsolved problem in the cognitive science of learning and instruction. In B.C. Love, K. McRae, \& V. M. Sloutsky (Eds.), Proceedings of the 30th Annual Conference of the Cognitive Science Society. (pp. 2155-2160).

Kumar, R., Rosé, C. P., Wang, Y. C., Joshi, M., \& Robinson, A. (2007). Tutorial dialogue as adaptive collaborative learning support. In R. Luckin, K. R. Koedinger, \& J. Greer (Eds.), Proceedings of artificial intelligence in education (pp. 383-390). IOS Press.

Magnisalis, I., Demetriadis, S., \& Karakostas, A. (2011). Adaptive and intelligent systems for collaborative learning support: a review of the field. IEEE Transactions on Learning Technologies, 4(1), 5-20.

Muldner, K., Burleson, W., \& VanLehn, K. (2010). "Yes!": Using tutor and sensor data to predict moments of delight during instructional activities. In P. DeBra, A. Kobsa, \& D. Chin (Eds.), Proceedings of the 18th International Conference on User Modeling, Adaptation and Personalization, UMAP (pp. 159-170). Berlin Heidelberg: Springer.

Nicol, D., \& Macfarlane-Dick, D. (2006). Formative assessment and self-regulated learning: a model and seven principles of good feedback practice. Studies in Higher Education, 31(2), 199-218.

Ogan, A., Aleven, V., Kim, J., \& Jones, C. (2010). Developing interpersonal relationships with virtual agents through social instructional dialog. In J. M. Allbeck, N. I. Badler, T. W. Bickmore, C. Pelachaud, \& A. Safonova (Eds.), Proceedings of the 10th International Conference on Intelligent Virtual Agents, IVA (pp. 236-249). Lecture Notes in Computer Science 6356. Berlin: Springer.

Prieto, L., Muñoz-Cristóbal, J., Asensio-Pérez, J., \& Dimitriadis, Y. (2012). Making Learning Designs Happen in Distributed Learning Environments with GLUE!-PS. In A. Ravenscroft, S. Lindstaedt, C. Kloos \& D. Hernández-Leo (Eds.), 21st Century Learning for 21st Century Skills (pp. 489494). Springer Berlin/Heidelberg.

Roll, I., Aleven, V., McLaren, B. M., \& Koedinger, K. R. (2011). Improving students help-seeking skills using metacognitive feedback in an intelligent tutoring system. Learning and Instruction, 21(2), 267-280.

Soller, A., Martínez, A., Jermann, P., \& Muehlenbrock, M. (2005). From mirroring to guiding: a review of state of the art technology for supporting collaborative learning. International Journal of Artificial Intelligence in Education, 15(4), 261-290.

Van den Bossche, P., Gijselaers, W. H., Segers, M., \& Kirschner, P. A. (2006). Social and cognitive factors driving teamwork in collaborative learning environments: team learning beliefs and behaviours. Small Group Research, 37, 490-521.

Walker, E., Rummel, N., \& Koedinger, K. (2009a). Beyond explicit feedback: new directions in adaptive collaborative learning support. In C. O’Malley, D. Suthers, P. Reimann, \& A. Dimitracopoulou (Eds.), Computer supported collaborative learning practices- CSCL 2009 conference proceedings, Vol 1 (pp. 552-556). International Society of the Learning Sciences, Inc.

Walker, E., Rummel, N., \& Koedinger, K. (2009b). CTRL: a research framework for providing adaptive collaborative learning support. User Modeling and User-Adapted Interaction: The Journal of Personalization Research (UMUAI), 19(5), 387-431.

Walker, E., Rummel, N., \& Koedinger, K. (2014). Adaptive intelligent support to improve peer tutoring in algebra. International Journal of Artificial Intelligence in Education, 24(1), 33-61. doi:10.1007/s40593-013-0001-9.

Wecker, C., \& Fischer, F. (2007). Fading scripts in computer-supported collaborative learning: the role of distributed monitoring. In C. Chinn, G. Erkens, \& S. Puntambekar (Eds.), Mice, minds, and society. Proceedings of the CSCL 2007. Computer supported collaborative learning (pp. 764-772). International Society of the Learning Sciences: Rutgers, The State University of New Jersey, New Brunswick, NJ.

Wecker, C., \& Fischer, F. (2011). From guided to self-regulated performance of domain-general skills: the role of peer monitoring during the fading of instructional scripts. Learning and Instruction, 21(6), 746-756. 